\title{
Organic Nonvolatile Memory Devices utilizing Intrinsic Charge-Trapping Phenomena in an n-Type Polymer Semiconductor
}

\author{
Nishit M. Murari, ${ }^{1}$ Ye-Jin Hwang, ${ }^{1}$ Felix Sunjoo Kim, ${ }^{2, *}$ Samson A. Jenekhe ${ }^{1, *}$ \\ 1 Department of Chemical Engineering and Department of Chemistry, University of \\ Washington, Seattle, WA 98195-1750, United States. \\ ${ }^{2}$ School of Chemical Engineering and Materials Science, Chung-Ang University, Seoul, 156- \\ 756, Republic of Korea \\ *E-mail: jenekhe@u.washington.edu; fskim@cau.ac.kr
}

Keywords: organic nonvolatile memory device, charge trapping, n-channel polymer transistor, switching phenomenon, polymer end capping

\begin{abstract}
Charge trapping is an undesirable phenomenon and a common challenge in the operation of n-channel organic field-effect transistors. Herein, we exploit charge trapping in an n-type semiconducting poly(naphthalene diimide biselenophene) (PNDIBS) as the key operational mechanism to develop high performance, nonvolatile, electronic memory devices. The PNDIBS-based field-effect transistor memory devices were programmed at $60 \mathrm{~V}$ and they showed excellent charge-trapping and de-trapping characteristics, which could be cycled more than 200 times with a current ratios of $10^{3}$ between the two binary states. Programmed data could be retained for $10^{3}$ seconds with a memory window of $28 \mathrm{~V}$. This is a record performance for $n$-channel organic transistor with inherent charge-trapping capability without using external charge trapping agents. However, the memory device performance was greatly reduced, as expected, when the n-type polymer semiconductor was end-capped with phenyl groups to reduce the trap density. These results show that the trap density of n-type semiconducting polymers could be engineered to control the inherent charge-trapping capability and device performance for developing high performance low cost memory devices.
\end{abstract}




\section{Introduction}

A low-cost approach of replacing conventional silicon devices with light-weight, highly flexible, organic electronic devices can enhance functionality and enable expanded applications.[1-7] This is especially true in the case of memory devices, wherein a greater degree of integration density is of perennial interest. Organic electronic memory has potential to combine high speed and high density with low power and low cost. Current organic memories based on capacitor and resistor architectures, while potentially useful in some applications, suffer from charge leakage, poor scaling, low device endurance, and low integration density, which have significantly restricted their potential commercial value. Another organic memory architecture for non-volatile data storage is based on the organic field-effect transistor (OFET), often with floating gates.[8] In this case, trapped charges in an OFET are switched between trapped and de-trapped states by applying an external voltage, giving stable low and high reading current levels which correspond to binary states of a memory. OFET-based memory is a promising candidate for future applications because of its easy compatibility with integrated circuits (ICs) and a possibility of three-dimensional stacking for ultra-high density memory storage of more than $10^{13} \mathrm{bits}_{\mathrm{in}}{ }^{2} \cdot[9,10]$

Most OFET memory devices reported to date are based on p-channel devices because of the ready availability of high-mobility $\left(\mu_{\mathrm{h}}>1 \mathrm{~cm}^{2} \mathrm{~V}^{-1} \mathrm{~s}^{-1}\right)$ p-type organic/polymer semiconductors.[11-13] The first reported p-channel OFET memory device used alphasexithiophene as the semiconductor with $\mathrm{SiO}_{2}$ and ferroelectric $\mathrm{PbZrTiO}_{3}(\mathrm{PZT})$ as gate dielectrics.[14] Later, p-type semiconducting polymers such as poly(selenophene-alt-3,6dithophene-2-yl-2,5-bis-(2-hexyldecyl)-2,5-dihydro-pyrrolo[3,4-c]pyrrole-1,4-dione)

(PSeDPP) were used to fabricate p-channel OFET memories.[15] All reported p-type semiconducting polymers for OFET memory devices suffer from low inherent charge trapping and retention capabilities. In order to address this problem, extra charge trapping 
agents such as metal nanoparticles or extra dielectric buffer layers were introduced in the device structure.[16] This helped in enhancing the charge trapping capability, retention and endurance. However, insertion of metal nanoparticles or a buffer layer in the structure introduces complexity in device fabrication and increases manufacturing time and cost.

n-Channel OFET-based memory devices are extremely rare because of the scarcity of high-mobility n-type semiconducting polymers. $[17,18]$ The first n-channel OFET memory device was based on fullerene (C60) with an electron-trapping polymer, poly(perfluoroalkenyl vinylether) (CYTOP).[19] This memory device could be programmed at $50 \mathrm{~V}$ for $0.1 \mathrm{~s}$ and gave a memory window of $10 \mathrm{~V}$. Recently, N,N'-bis(2-phenylethyl) perylene-3,4:9,10-tetracarboxylic diimide (BPE-PTCDI) and polyimide electrets, such as poly[2,5-bis(4-aminophenylenesulfanyl)selenophene-hexafluoroisopropylidene diphthalimide] (PI(APSP-6FDA)), poly-[2,5-bis(4-aminophenylenesulfanyl)thiophenehexafluoroisopropylidene diphthalimide] (PI(APST-6FDA)), and poly(4,4'-oxidianiline-4,4'hexafluoroisopropylidenediphthalic anhydride) (PI(ODA-6FDA)), were used for charge trapping.[20, 21] The n-type polymer, poly $\{[\mathrm{N}, \mathrm{N} 0-\mathrm{bis}(2-$ octyldodecyl)-naphthalene-1,4,5,8bis(dicarboximide)-2,6-diyl]-alt-5,50-(2,20-bithiophene)\} [P(NDI2OD-T2)], has also been used in OFET memory devices; however, the device performance improved significantly only after using an additional poly(3-hexylthiophene) (P3HT) film on a poly(methylmethacrylate) (PMMA) dielectric layer for trapping charges.[22]

OFET memory devices without any additional charge-trapping components do not have sufficient amount of charge trapping for two stable memory states. This low chargetrapping capability decreases the device performance and limits the commercial viability of OFET memory devices. The discovery of high-performance n-type polymer semiconductors with inherent charge-trapping capability would represent a major step towards commercially viable organic electronic memory technology. Devices fabricated using such high-mobility n- 
type semiconducting polymers could facilitate the development of low cost ultra-high density memories.

Our group reported the first n-channel polymer OFETs, achieving a field-effect electron mobility of $0.1 \mathrm{~cm}^{2} \mathrm{~V}^{-1} \mathrm{~s}^{-1}$ based on poly(benzimidazobenzophenanthroline ladder)(BBL).[23] A perylenediimide (PDI)-arylene copolymer was later reported with a mobility of $0.013 \mathrm{~cm}^{2} \mathrm{~V}^{-1} \mathrm{~s}^{-1}$.[24] PDI and its structural analog, naphthalenediimide (NDI)based polymers, are among the most extensively investigated n-type organic semiconductors.[25-27] NDI-based conjugated polymer semiconductors are attractive because of their easy synthesis, better solubility, and increased crystallinity compared to PDIbased conjugated polymers. Recently, our group showed that a high field-effect electron mobility of $0.24 \mathrm{~cm}^{2} \mathrm{~V}^{-1} \mathrm{~s}^{-1}$ can be achieved from copolymers of naphthalene diimide and various selenophene derivatives.[28, 29] High-mobility n-type semiconducting polymers could enable fast-switching electronic circuits and memories.[30]

In this paper, we report high performance, solution-processed, n-channel OFET memory devices based on the high-mobility n-type polymer semiconductor, poly $\{[\mathrm{N}, \mathrm{N}$ 'bis(2-decyltetradecyl)-naphthalene-1,4,5,8-bis(dicarboximide)-2,6-diyl]-alt-5,5' -(2,2'biselenophene)\} (PNDIBS) (Figure 1). The inherent charge-trapping capability of PNDIBS showed stability of more than 200 switching cycles with a consistent on/off current ratio of more than two orders of magnitude when programmed at a low gate bias of $60 \mathrm{~V}$ applied for 1 second. Excellent data retention of $10^{3}$ seconds with a $10^{3}$ to $10^{4}$ on/off current ratio makes these the best-performing OFET memory devices reported so far. We also observed that OFET memory devices fabricated from phenyl end-capped derivative of PNDIBS (ePNDIBS) showed overall lower device performance in comparison to PNDIBS. The memory window was reduced from $28 \mathrm{~V}$ in PNDIBS to $17 \mathrm{~V}$ in the ePNDIBS while the charge retention capability also decreased by an order of magnitude. The switching cycle of 
ePNDIBS devices showed a consistent reduction in the high current state and on/off current ratio. Dielectric spectroscopy reveals a significant decrease in the trap density of ePNDIBS compared to PNDIBS without phenyl end-capping. The decrease in the trap density reduces the amount of electrons involved in trapping and de-trapping, which thereby reduces the OFET memory device performance. Our results show that electron trapping, which is seen as a common problem in the development and operation of n-channel OFETs, can be significantly tuned by engineering the polymer structure and exploited to create useful highperformance electronic memory devices.

\section{Experimental Section}

Materials: The synthesis and characterization of the n-channel polymer semiconductors, PNDIBS and ePNDIBS, have been previously reported.[28, 29] Samples of the two polymers used in the present study had number average molecular weight $\left(M_{n}\right)$ of $42.4 \mathrm{~kg} / \mathrm{mol}$ for PNDIBS and $40.1 \mathrm{~kg} / \mathrm{mol}$ for ePNDIBS and both have a polydispersity index (PDI) of 2.6. The field-effect electron mobilities of the PNDIBS and ePNDIBS are 0.07 and $0.24 \mathrm{~cm}^{2} \mathrm{~V}^{-1} \mathrm{~s}^{-1}$, respectively.

Memory Device Fabrication and Testing: The memory devices based on PNDIBS and ePNDIBS were fabricated as a bottom-gate/top-contact (BGTC) OFET structure on a heavily n-doped $<100>$ silicon wafer $(0.002-0.004 \Omega / \mathrm{cm})$ which served as the gate electrode while its $200 \mathrm{~nm}$ thermally grown $\mathrm{SiO}_{2}$ layer served as the gate dielectric $\left(\mathrm{C}_{\mathrm{ox}}=17 \mathrm{nF} / \mathrm{cm}^{2}\right)$. The wafer was cleaned by sequential ultra-sonication in acetone and isopropanol followed by plasma treatment. Then, the wafer surface was modified by formation of an octyltrichlorosilane (OTS-8) self-assembled monolayer by spin coating a $1 \mathrm{mM}$ solution of OTS- 8 in chloroform at $3000 \mathrm{rpm}$ for 10 seconds, rinsed with toluene and dried at $100^{\circ} \mathrm{C}$ for 10 minutes in air. Each polymer (PNDIBS or ePNDIBS) thin film was deposited onto the OTS-8 modified substrate by spin coating a polymer solution $(8 \mathrm{mg} / \mathrm{mL})$ in chloroform at 
$2000 \mathrm{rpm}$ for 60 seconds followed by annealing at $200^{\circ} \mathrm{C}$ for 10 minutes on a hot plate under argon atmosphere. The silver source/drain electrodes were deposited by vacuum evaporation through a shadow mask to define a transistor channel (length/width of $100 \mu \mathrm{m} / 1000 \mu \mathrm{m}$ ) (Figure 1). All the electrical characteristics of the OFET memory devices were measured by using an HP 4145B semiconductor parameter analyzer in the nitrogen atmosphere. Capacitance measurement was performed by using an HP 4284A LCR meter.

\section{Results and Discussion}

The writing and erasing characteristics of PNDIBS- and ePNDIBS-based OFET memory devices are shown in Figure 2. The transfer curves, i.e. plots of drain current as a function of gate voltage, of the memory devices significantly shifted to a very negative gate bias after applying a writing gate voltage pulse of $-60 \mathrm{~V}$ for 1 second. The direction of the threshold voltage shift coincides with the polarity of the applied writing pulse through the gate electrode, indicating trapping and detrapping events of charges in the bulk polymer semiconductor thin film and at the polymer/dielectric interface.[31] Memory devices based on PNDIBS showed a shift in threshold voltage from $6 \mathrm{~V}$ to $-22 \mathrm{~V}$ while ePNDIBS had a shift from $3 \mathrm{~V}$ to $-14 \mathrm{~V}$. Subsequent application of an erasing pulse of reverse gate bias voltage of $60 \mathrm{~V}$ for 1 second caused the transfer curve to retrace back to the initial position. This shift in transfer curve with writing and erasing pulses created a memory window of $28 \mathrm{~V}$ for PNDIBS and $17 \mathrm{~V}$ for ePNDIBS. The reduced memory window of ePNDIBS-based devices, in comparison of PNDIBS devices, indicates a reduced amount of sites available for trapping and detrapping of electrons. This shows that end-capping of the polymer semiconductor affects the device performance by tuning the inherent charge-trapping capability of the n-channel material. The threshold voltage of PNDIBS (or ePNDIBS) did not shift beyond the initial position even after applying a larger positive bias. This shift in the transfer curves, caused by a single type of charge-carriers, produces two distinct values of 
high and low current at the zero gate voltage. These two states of drain current represent two logic values, 1 and 0 , respectively, which form the basis for data storage in the OFET-based memory devices.

Figure 3 shows that the shift in the transfer curve follows a Boltzmann function with two distinct threshold voltages. The transfer curve measured at different gate voltages showed an exponential increase and saturate at $-60 \mathrm{~V}$. The solid line shows the shift in threshold voltage fitted with the Boltzmann function given by:

$$
V_{T h}=V_{2}+\frac{V_{1}-V_{2}}{1+e^{\left(V_{g}-V_{3}\right) / V_{0}}}
$$

The least-square fitting gives excellent agreement with the voltage parameters $V_{1}, V_{2}, V_{3}$ and $\mathrm{V}_{0}$ as $30.4,4.8,-45.0$, and $7.7 \mathrm{~V}$, respectively. This result shows an interesting and important phenomenon, there is a negligible shift of transfer curve at low programming voltages and consequently allows for a nondestructive read-out. The high turn-on voltage after programming results in the large difference between two drain current levels in the $\mathrm{ON}$ and OFF states. This makes it a better performing memory device in terms of noise immunity.

To measure the lifetime of the stored data, or in other words the non-volatility of the memory, we performed a retention test (or Write-Once-Read-Many-times (WORM) measurement) of PNDIBS and ePNDIBS memory devices as a function of time (Figure 4). The current decay of the $\mathrm{ON}$-state was performed by measuring drain current with respect to time after applying a writing pulse of $\mathrm{V}_{\mathrm{gs}}=-60 \mathrm{~V}$ for 1 second. An erasing pulse of $\mathrm{V}_{\mathrm{gs}}=60 \mathrm{~V}$ was applied for the decay of OFF-state. The two distinct current values shown as ON- and OFF-states are used to implement binary logic level (1 and 0) in the memory device which can be set and reset by applying negative and positive gate voltage pulses $\left(\mathrm{V}_{\mathrm{gs}}= \pm 60 \mathrm{~V}\right)$. The retention of data in the PNDIBS based memory devices (Figure 4a) maintains the difference 
between high and low conducting states for more than 800 seconds with some initial decay. The ON/OFF current ratio is maintained in the range of $10^{3}-10^{4}$ at a read voltage of $0 \mathrm{~V}$. This consistent difference between ON- and OFF-state current levels for a long time of more than 800 second represents an excellent reliability of organic memory devices in storing data. Figure 4(b) shows the retention test (WORM) of an ePNDIBS-based memory device. The ON-current decayed by an order of magnitude within 400 seconds while the OFF-current decayed by two orders of magnitude within 100 seconds and then stabilized. The overall on/off ratio was of the order of $10^{2}$. PNDIBS-based memory devices can retain data at two binary states with a larger current difference for a longer time compared to ePNDIBS-based devices, implying that the PNDIBS-based memory devices are more stable and reliable in terms of data retention.

In order to understand the decay behavior of drain current from the retention test, we used an exponential decay analysis method. An exponential decay curve with three time constants was used for this analysis. The decay time constants were extracted by fitting the drain current using an exponential decay equation:

$$
I_{\mathrm{D}}=I_{0}+A_{1} e^{-t / t_{1}}+A_{2} e^{-t / t_{2}}+A_{3} e^{-t / t_{3}}
$$

where $I_{0}$ is the base current, $A_{1}, A_{2}$, and $A_{3}$ are the pre-exponential factors, and $t_{1}, t_{2}$ and $t_{3}$ are the time constants. Although, a drain current decay generally follows an exponential trend with two time constants which corresponds to the two energy levels of interfacial traps,[32] we observed that, in our case, two time constants were not enough to achieve a good fit; a better fit was achieved by using three time constants.[33] The absolute value of three time constants of ON and OFF state for PNDIBS memory devices extracted by fitting the drain current from retention measurement, as shown in Figure 4(a), were 1.9, 19.2, and 208.0 seconds and 0.7, 6.5, and 66.8 seconds, respectively. The corresponding pre-exponential 
factors were $2.14 \times 10^{-7}, 9.87 \times 10^{-8}$, and $2.77 \times 10^{-8} \mathrm{~A}$ for ON-state, and $1.73 \times 10^{-9}, 3.43 \times 10^{-10}$, and $4.97 \times 10^{-11} \mathrm{~A}$ for OFF-state. The ON state time constants are three times higher than the OFF state, which explains the long retention with a high ratio of ON and OFF current levels. The decay current for ePNDIBS also gave an excellent fit with exponential decay by using three time-constants as shown in Figure 4(b). Three time constants for ON- and OFF states are 2.3, 24.0, 222.6 and 0.7, 4.4, and 21.5 seconds respectively. Unlike PNDIBS, the ratios of time constants for ON and OFF-state of ePNDIBS vary from 3.3 to 10.4. The corresponding pre-exponential factors for ePNDIBS are $1.45 \times 10^{-7}, 8.83 \times 10^{-8}$, and $2.75 \times 10^{-8} \mathrm{~A}$, respectively, for the ON state, $2.55 \times 10^{-8}, 1.06 \times 10^{-8}$, and $2.93 \times 10^{-9} \mathrm{~A}$, respectively, for the OFF state. The memory transistor decay current is likely affected by the charges stored at the interfaces and in the bulk polymer film. The first two exponential components of equation (2) are related to charges stored at the interfaces, which are easily neutralized by ions at the interfaces and thus correspond to the shorter time constants. The third component is related to charges stored in the bulk polymer film, which are more stable and give rise to the longer time constant. Hence, we see a longer bulk retention time in the end-capped polymer.

The endurance stability of the devices, measured by a multi-cycling test of repeated Write-Read-Erase-Read (WRER) sequence, is shown in Figure 5. The writing and erasing of the memory devices were set by the gate bias voltages of $\pm 60 \mathrm{~V}$ at a drain-source voltage $\mathrm{V}_{\mathrm{ds}}=0 \mathrm{~V}$, while the reading is done at $\mathrm{V}_{\mathrm{gs}}=0 \mathrm{~V}$ and $\mathrm{V}_{\mathrm{ds}}=40 \mathrm{~V}$. PNDIBS-OFET memory devices showed excellent endurance with consistent reversible and stable memory-cycling behavior for more than 200 cycles as shown in Figure 5(a). The on/off current ratio remained higher than $10^{2}$ throughout the switching cycles. The consistency of PNDIBS high conducting state after extensive WRER testing, which involves trapping and de-trapping of charges, demonstrates that there is no reduction or loss of electrons. Thus, our PNDIBS-based charge-trapping OFET memory is advantageous because of the low powered non-destructive 
device operation with much higher electrical stability than any other reported OFET memory device. Figure 5(b) shows the enlarged view of the WRER cycle.

For comparison, the WRER endurance measurement of ePNDIBS-OFET is also plotted in Figure 5(c). Noticeable decay of ON-current in the ePNDIBS devices under repeated cycling at the same conditions was observed. The decrease in ON-current by one order of magnitude within 200 cycle shows that the endurance of the memory device is substantially reduced by the end-capping of the polymer semiconductor. Extrapolation of the data suggests that the ON-current of ePNDIBS would reduce to the OFF-current level within 1000 cycles, while a similar projection of the ON/OFF ratio of PNDIBS is high $\left(>10^{2}\right)$ and stable for more than 1000 cycles. Highly reliable data retention and endurance of PNDIBS in comparison to ePNDIBS is remarkable, considering that such a small change in the polymer chain ends can have a large impact in data retention and switching endurance. Our present results clearly demonstrate that the OFET memory device performance can be tuned and precisely controlled by small changes in the polymer structures. Such a molecular-level engineering strategy opens up a new direction for the development of high-performance organic memory devices.

In order to characterize the trap density and understand the kinetics of trapped charges in PNDIBS and ePNDIBS, we performed capacitance and conductance measurements at different frequencies. The measurements were carried out in the frequency range $24 \mathrm{~Hz}-1$ $\mathrm{MHz}$ with bias from $\mathrm{V}=-40$ to $+40 \mathrm{~V}$ with oscillator voltage level of $0.025 \mathrm{~V}$. The $\mathrm{C}-\mathrm{V}$ curves in Figure 6(a) represent a typical n-type behavior with a high-capacitance accumulation region under the positive bias and a low-capacitance depletion region under the negative bias. After saturation in the accumulation and depletion regions, the capacitance becomes constant and independent of bias voltage and frequency. The capacitance appears constant in the accumulation region for both polymers and then decreases sharply in the 
voltage region between 21 and $-2 \mathrm{~V}$ for the PNDIBS and 16 and $5 \mathrm{~V}$ for the ePNDIBS. As discussed earlier, the transfer curves of PNDIBS-OFETs formed a larger hysteresis window of $28 \mathrm{~V}$ in comparison with ePNDIBS-OFETs, which formed a window of $17 \mathrm{~V}$. During the bias sweeping, PNDIBS showed a higher capacitance density and larger turn-on voltage than ePNDIBS. Turn-on voltage is defined as the gate voltage below which the transistor is offstate.

The increase in capacitance density and turn-on voltage can be explained by the amount of traps present in the semiconductor.[34] Energetic disorder, due mainly to structural inhomogeneities and defects or chemical impurities, give rise to charge-trapping sites in polymer semiconductors. These inhomogeneities or impurities may be generated in a polymer by virtue of chemical defects such as chain ends, breaks, twists, or thermo-, photo-, or electro-oxidative degradation, and even a very small fraction of such disorders can have a large impact on the electronic properties.[35-37] These trapping sites can be addressed and charge injection and transport can be significantly improved by elimination of such sites through end-capping of the polymer chains. Phenyl end-capping provides high stability against oxidation and may have prevented structural defects such as twisting of the aromatic chain backbone and coiling in polymer chains which are known to create high trap densities. ${ }^{[38-40]}$ The highest occupied molecular orbitals/lowest unoccupied molecular orbitals are $-5.95 \mathrm{eV} /-3.94 \mathrm{eV}$ for PNDIBS and $-5.90 \mathrm{eV} /-3.90 \mathrm{eV}$ for ePNDIBS based on cyclic voltammetry, implying that the changes in the energy levels may not be the main cause of trapping events. However, as shown in the absorption spectra of the polymers (Figure S1), a higher intensity of intramolecular charge transfer (ICT) band (500-900 nm) in ePNDIBS is seen compared with that in PNDIBS. This indicates stronger interaction between polymer chains in a thin film of ePNDIBS, due likely to the highly organized packing structure of ePNDIBS. 
It has been suggested that the presence of electrons and trap recharging may result in the evolution of hysteresis.[41] It can be concluded that PNDIBS without end-capping has a higher amount of traps present which increases the amount of electrons trapped in the bulk and at the interface compared to the end-capped ePNDIBS. Increased amount of trapped charges increases the capacitance and reduces the turn-on voltage. Because extra charge carriers are required to fill the traps, it takes more total charge-carriers and a larger applied voltage to accomplish the same surface potential (or band bending). We also observed that there was no change in the shape of the $\mathrm{C}-\mathrm{V}$ curves by end-capping of the polymer. This confirms that the origin of the $\mathrm{C}-\mathrm{V}$ stretching is trapped charges since the charged traps in the semiconductor near the interface would simply increase the charge density in the depletion region, causing the $\mathrm{C}-\mathrm{V}$ plot to stretch to higher voltages.[42]

Frequency dispersion (FD) of the capacitance in the accumulation region was observed for both the polymers as shown in Figure 6(b). The FD of PNDIBS at the gate bias of $-40 \mathrm{~V}$ is clearly stronger compared with ePNDIBS. Capacitance FD is due to the different responses of trapped electrons at shallow and deep levels and depends on the trapping more than the de-trapping of charge-carriers in organic semiconductors.[43] The accumulation capacitance decreased with increasing frequency, indicating the presence of deep states with long time constant. The high capacitance observed at lower frequency suggests that electrons at lower frequencies are likely to be the trapped charges. ${ }^{[44]}$ In order to calculate the concentration of traps we used the linear section of the $1 / C^{2}$ vs V plot with the Schottky-Mott equation: $^{[45]}$

$$
\frac{\delta\left(C^{-2}\right)}{\delta V}=\frac{2}{q \varepsilon_{0} \varepsilon_{r} N}
$$

where $\mathrm{C}$ is the capacitance density in the depletion region, $\mathrm{V}$ is the gate bias, $\varepsilon_{0}$ is the permittivity of free space, $\varepsilon_{\mathrm{r}}$ is the relative dielectric constant of the semiconductor, and $\mathrm{N}$ is 
the concentration of the localized charges. The concentration of traps in PNDIBS and ePNDIBS was calculated to be $4.0 \times 10^{17} \mathrm{~cm}^{-3}$ and $2.1 \times 10^{17} \mathrm{~cm}^{-3}$ at $1 \mathrm{MHz}$, respectively, showing that the density of traps present in PNDIBS is two times higher than that in ePNDIBS. This means that the amount of traps in the polymer semiconductor is reduced by half by phenyl end-capping. These results suggest that molecular engineering can provide a means of tuning the intrinsic charge-trapping sites in a polymer semiconductor to enable high-performance memory devices with a simple architecture.

\section{Conclusions}

Our study has exploited the normally undesirable charge trapping in n-type semiconducting polymers as a basis for developing high performance OFET memory devices. The n-type OFET memory devices were fabricated using the high-mobility n-type polymer, PNDIBS, with inherent charge-trapping capacity. These n-type PNDIBS-OFET memory devices combined endurance of more than 200 cycles with data retention of $10^{3}$ second and over $10^{3}$ on/off current level difference between the two binary memory states, which represent the best performance seen so far among OFET memory devices. Similarly fabricated OFET memory devices based on the phenyl end-capped ePNDIBS showed a much lower device performance in agreement with the lower trap density and lower chargetrapping capability observed in capacitance-voltage measurements. These results demonstrate that high-performance OFET memory devices can be fabricated by utilizing intrinsic traps present in n-type polymer semiconductors.

\section{Acknowledgements}

This work was supported by the NSF (DMR-1409687) and the Boeing-Martin Professorship. F.S.K. acknowledges financial support from the National Research Foundation of Korea grant funded by the Korea Government (NRF-2014M3A7B4051749 and NRF2014R1A1A2058289). 


\section{Notes and references}

[1] Y.-H. Chou, H.-C. Chang, C.-L. Liu, W.-C. Chen, Polymeric charge storage electrets for non-volatile organic field effect transistor memory devices, Polym. Chem., 6 (2015) 341-352. [2] P. Heremans, G.H. Gelinck, R. Müller, K.-J. Baeg, D.-Y. Kim, Y.-Y. Noh, Polymer and Organic Nonvolatile Memory Devices, Chem. Mater., 23 (2011) 341-358.

[3] C.-L. Liu, W.-C. Chen, Donor-acceptor polymers for advanced memory device applications, Polym. Chem., 2 (2011) 2169-2174.

[4] R.C.G. Naber, K. Asadi, P.W.M. Blom, D.M. de Leeuw, B. de Boer, Organic Nonvolatile Memory Devices Based on Ferroelectricity, Adv. Mater., 22 (2010) 933-945.

[5] J. Ouyang, C.-W. Chu, C.R. Szmanda, L. Ma, Y. Yang, Programmable polymer thin film and non-volatile memory device, Nat. Mater., 3 (2004) 918-922.

[6] J.C. Scott, L.D. Bozano, Nonvolatile Memory Elements Based on Organic Materials, Adv. Mater., 19 (2007) 1452-1463.

[7] M.S. Choi, G.-H. Lee, Y.-J. Yu, D.-Y. Lee, S.H. Lee, P. Kim, J. Hone, W.J. Yoo, Controlled charge trapping by molybdenum disulphide and graphene in ultrathin heterostructured memory devices, Nat. Commun., 4 (2013) 1624.

[8] T. Sekitani, T. Yokota, U. Zschieschang, H. Klauk, S. Bauer, K. Takeuchi, M. Takamiya, T. Sakurai, T. Someya, Organic Nonvolatile Memory Transistors for Flexible Sensor Arrays, Science, 326 (2009) 1516-1519.

[9] S. Song, B. Cho, T.W. Kim, Y. Ji, M. Jo, G. Wang, M. Choe, Y.H. Kahng, H. Hwang, T. Lee, Three-Dimensional Integration of Organic Resistive Memory Devices, Adv. Mater., 22 (2010) 5048-5052.

[10] Y.-W. Lin, C.-J. Lin, Y.-H. Chou, C.-L. Liu, H.-C. Chang, W.-C. Chen, Nonvolatile organic field effect transistor memory devices using one-dimensional aligned electrospun nanofiber channels of semiconducting polymers, J. Mater. Chem. C, 1 (2013) 5336-5343. 
[11] J.S. Ha, K.H. Kim, D.H. Choi, 2,5-Bis(2-octyldodecyl)pyrrolo[3,4-c]pyrrole-1,4(2H,5H)-dione-Based Donor-Acceptor Alternating Copolymer Bearing 5,5' -Di(thiophen-2yl)-2,2' -biselenophene Exhibiting $1.5 \mathrm{~cm} 2 \cdot \mathrm{V}-1 \cdot \mathrm{s}-1$ Hole Mobility in Thin-Film Transistors, J. Am. Chem. Soc., 133 (2011) 10364-10367.

[12] W. Zhang, J. Smith, S.E. Watkins, R. Gysel, M. McGehee, A. Salleo, J. Kirkpatrick, S. Ashraf, T. Anthopoulos, M. Heeney, I. McCulloch, Indacenodithiophene Semiconducting Polymers for High-Performance, Air-Stable Transistors, J. Am. Chem. Soc., 132 (2010) 11437-11439.

[13] G. Kim, S.-J. Kang, G.K. Dutta, Y.-K. Han, T.J. Shin, Y.-Y. Noh, C. Yang, A Thienoisoindigo-Naphthalene Polymer with Ultrahigh Mobility of $14.4 \mathrm{~cm} 2 / \mathrm{V} \cdot \mathrm{s}$ That Substantially Exceeds Benchmark Values for Amorphous Silicon Semiconductors, J. Am. Chem. Soc., 136 (2014) 9477-9483.

[14] G. Velu, C. Legrand, O. Tharaud, A. Chapoton, D. Remiens, G. Horowitz, Low driving voltages and memory effect in organic thin-film transistors with a ferroelectric gate insulator, Appl. Phys. Lett., 79 (2001) 659-661.

[15] H.-W. Lin, W.-Y. Lee, W.-C. Chen, Selenophene-DPP donor-acceptor conjugated polymer for high performance ambipolar field effect transistor and nonvolatile memory applications, J. Mater. Chem., 22 (2012) 2120-2128.

[16] X.-J. She, C.-H. Liu, Q.-J. Sun, X. Gao, S.-D. Wang, Morphology control of tunneling dielectric towards high-performance organic field-effect transistor nonvolatile memory, Org. Electron., 13 (2012) 1908-1915.

[17] S. Allard, M. Forster, B. Souharce, H. Thiem, U. Scherf, Organic semiconductors for solution-processable field-effect transistors (OFETs), Angew. Chem. Int. Ed., 47 (2008) 4070-4098. 
[18] J. Zaumseil, H. Sirringhaus, Electron and ambipolar transport in organic field-effect transistors, Chem. Rev., 107 (2007) 1296-1323.

[19] T.T. Dao, T. Matsushima, H. Murata, Organic nonvolatile memory transistors based on fullerene and an electron-trapping polymer, Org. Electron., 13 (2012) 2709-2715.

[20] Y.-H. Chou, N.-H. You, T. Kurosawa, W.-Y. Lee, T. Higashihara, M. Ueda, W.-C. Chen, Thiophene and Selenophene Donor-Acceptor Polyimides as Polymer Electrets for Nonvolatile Transistor Memory Devices, Macromolecules, 45 (2012) 6946-6956.

[21] Y.-H. Chou, W.-Y. Lee, W.-C. Chen, Self-Assembled Nanowires of Organic n-Type Semiconductor for Nonvolatile Transistor Memory Devices, Adv. Funct. Mater., 22 (2012) $4352-4359$.

[22] K.A. Mohamad, K. Yousuke, K. Uesugi, H. Fukuda, n-Channel Organic Thin-Film Transistors based on Naphthalene-Bis(dicarboximide) Polymer for Organic Transistor Memory Using Hole-Acceptor Layer, Jpn. J. Appl. Phys., 50 (2011) 091603.

[23] A. Babel, S.A. Jenekhe, High electron mobility in ladder polymer field-effect transistors, J. Am. Chem. Soc., 125 (2003) 13656-13657.

[24] X. Zhan, Z. Tan, B. Domercq, Z. An, X. Zhang, S. Barlow, Y. Li, D. Zhu, B. Kippelen, S.R. Marder, A High-Mobility Electron-Transport Polymer with Broad Absorption and Its Use in Field-Effect Transistors and All-Polymer Solar Cells, J. Am. Chem. Soc., 129 (2007) 7246-7247.

[25] F.S. Kim, X. Guo, M.D. Watson, S.A. Jenekhe, High-mobility Ambipolar Transistors and High-gain Inverters from a Donor-Acceptor Copolymer Semiconductor, Adv. Mater., 22 (2010) 478-482.

[26] Z. Chen, Y. Zheng, H. Yan, A. Facchetti, Naphthalenedicarboximide- vs Perylenedicarboximide-Based Copolymers. Synthesis and Semiconducting Properties in Bottom-Gate N-Channel Organic Transistors, J. Am. Chem. Soc., 131 (2009) 8-9. 
[27] X. Guo, F.S. Kim, M.J. Seger, S.A. Jenekhe, M.D. Watson, Naphthalene Diimide-Based Polymer Semiconductors: Synthesis, Structure-Property Correlations, and n-Channel and Ambipolar Field-Effect Transistors, Chem. Mater., 24 (2012) 1434-1442.

[28] Y.-J. Hwang, N.M. Murari, S.A. Jenekhe, New n-type polymer semiconductors based on naphthalene diimide and selenophene derivatives for organic field-effect transistors, Polym. Chem., 4 (2013) 3187-3195.

[29] Y.-J. Hwang, G. Ren, N.M. Murari, S.A. Jenekhe, n-Type Naphthalene DiimideBiselenophene Copolymer for All-Polymer Bulk Heterojunction Solar Cells, Macromolecules, 45 (2012) 9056-9062.

[30] H. Li, F.S. Kim, G. Ren, S.A. Jenekhe, High-Mobility n-Type Conjugated Polymers Based on Electron-Deficient Tetraazabenzodifluoranthene Diimide for Organic Electronics, J. Am. Chem. Soc., 135 (2013) 14920-14923.

[31] K.-J. Baeg, D. Khim, J. Kim, B.-D. Yang, M. Kang, S.-W. Jung, I.-K. You, D.-Y. Kim, Y.-Y. Noh, High-Performance Top-Gated Organic Field-Effect Transistor Memory using Electrets for Monolithic Printed Flexible NAND Flash Memory, Adv. Funct. Mater., 22 (2012) 2915-2926.

[32] R.-I. Yamada, J. Yugami, Analysis of the exponentially decaying transient current in MOS capacitors, 40th Annual Reliability Physics Symposium Proceedings, (2002) 387-392.

[33] A.D. Bartolomeo, M. Rinzan, A.K. Boyd, Y. Yang, L. Guadagno, F. Giubileo, P. Barbara, Electrical properties and memory effects of field-effect transistors from networks of single- and double-walled carbon nanotubes, Nanotechnology, 21 (2010) 115204.

[34] H.T. Nicolai, M. Kuik, G.A.H. Wetzelaer, B. de Boer, C. Campbell, C. Risko, J.L. Brédas, P.W.M. Blom, Unification of trap-limited electron transport in semiconducting polymers, Nat. Mater., 11 (2012) 882-887. 
[35] J.K. Park, J. Jo, J.H. Seo, J.S. Moon, Y.D. Park, K. Lee, A.J. Heeger, G.C. Bazan, EndCapping Effect of a Narrow Bandgap Conjugated Polymer on Bulk Heterojunction Solar Cells, Adv. Mater., 23 (2011) 2430-2435.

[36] Q. Wang, B. Zhang, L. Liu, Y. Chen, Y. Qu, X. Zhang, J. Yang, Z. Xie, Y. Geng, L. Wang, F. Wang, Effect of End Groups on Optoelectronic Properties of Poly(9,9dioctylfluorene): A Study with Hexadecylfluorenes as Model Polymers, J. Phys. Chem. C, 116 (2012) 21727-21733.

[37] M.J. Robb, D. Montarnal, N.D. Eisenmenger, S.-Y. Ku, M.L. Chabinyc, C.J. Hawker, A One-Step Strategy for End-Functionalized Donor-Acceptor Conjugated Polymers, Macromolecules, 46 (2013) 6431-6438.

[38] A.M. Funston, E.E. Silverman, J.R. Miller, K.S. Schanze, Charge Transfer through Terthiophene End-Capped Poly(arylene ethynylene)s, J. Phys. Chem. B, 108 (2004) 15441555.

[39] A. Kadashchuk, R. Schmechel, H. von Seggern, U. Scherf, A. Vakhnin, Charge-carrier trapping in polyfluorene-type conjugated polymers, J. Appl. Phys., 98 (2005) 024101.

[40] W. Wang, A.G. MacDiarmid, New synthesis of phenyl/phenyl end-capped tetraaniline in the leucoemeraldine and emeraldine oxidation states, Synth. Met., 129 (2002) 199-205.

[41] T. Lindner, G. Paasch, S. Scheinert, Hysteresis in organic field-effect devices: Simulated effects due to trap recharging, J. Appl. Phys., 98 (2005) 114505.

[42] D.M. Taylor, Space charges and traps in polymer electronics, Dielectrics and Electrical Insulation, IEEE Transactions on, 13 (2006) 1063-1073.

[43] P.J. Brown, H. Sirringhaus, M. Harrison, M. Shkunov, R.H. Friend, Optical spectroscopy of field-induced charge in self-organized high mobility poly(3-hexylthiophene), Phys. Rev. B, 63 (2001) 125204. 
[44] M. Yun, R. Ravindran, M. Hossain, S. Gangopadhyay, U. Scherf, T. Bünnagel, F. Galbrecht, M. Arif, S. Guha, Capacitance-voltage characterization of polyfluorene-based metal-insulator-semiconductor diodes, Appl. Phys. Lett., 89 (2006) 013506.

[45] S.M. Sze, K.K. Ng, Physics of semiconductor devices, 3rd ed., John Wiley \& Sons, New York, 2007. 


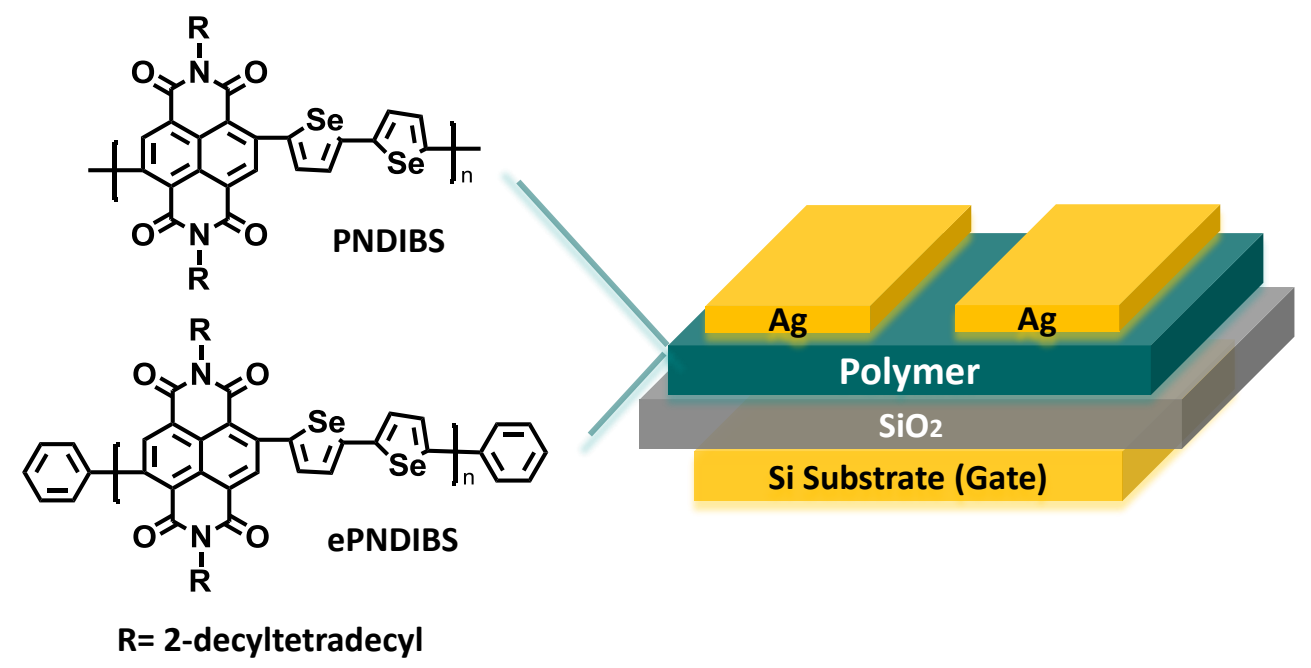

Figure 1. Molecular structures of the n-type polymer semiconductors (PNDIBS and ePNDIBS) investigated and schematic of the charge-trapping organic field-effect transistor memory devices. 

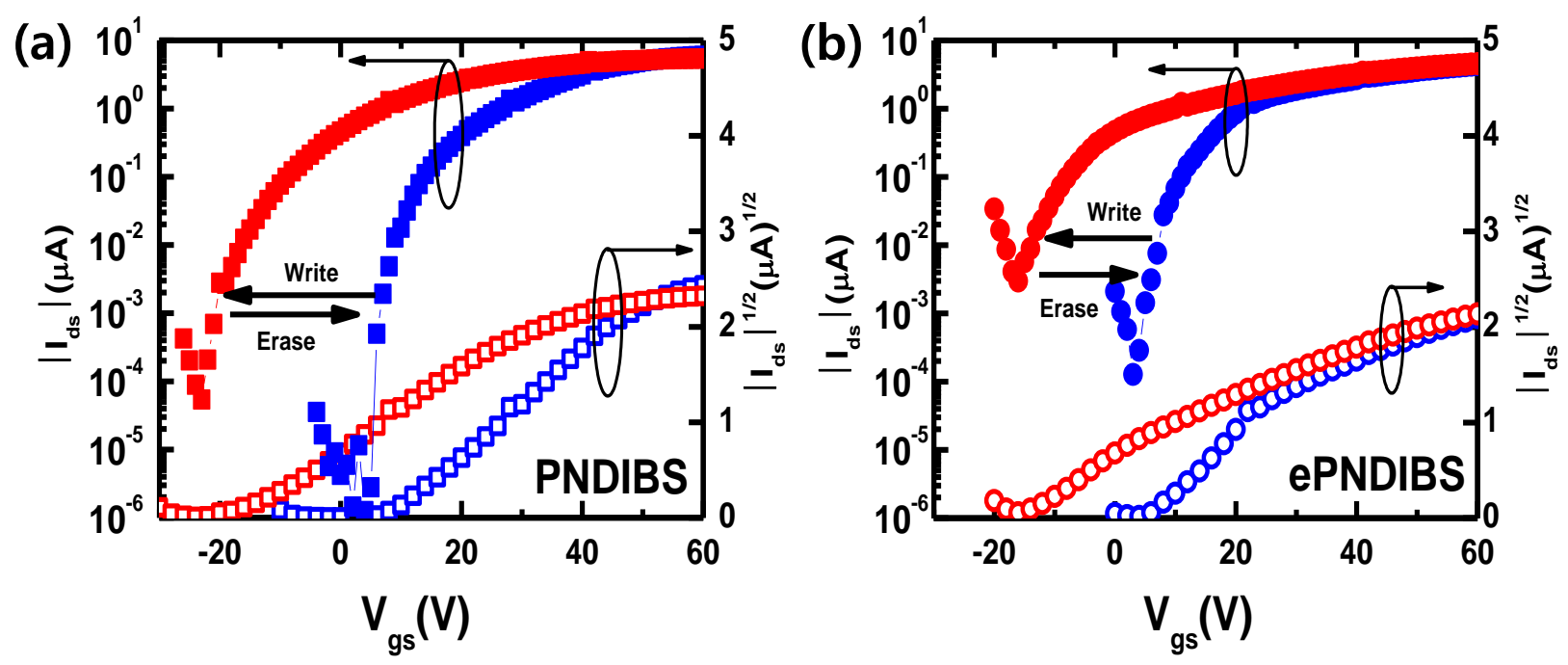

Figure 2. Transfer curves for the transistor memory devices of (a) PNDIBS and (b) ePNDIBS. Gate voltages, $\mathrm{V}_{\mathrm{gs}}$, of $-60 \mathrm{~V}$ and $+60 \mathrm{~V}$ were applied for 1 second for writing and erasing, respectively. Drain voltage, $\mathrm{V}_{\mathrm{ds}}$, was $60 \mathrm{~V}$. The red curve represents high $(\mathrm{ON})$ state while blue represents low (OFF) conducting state of the memory devices. 


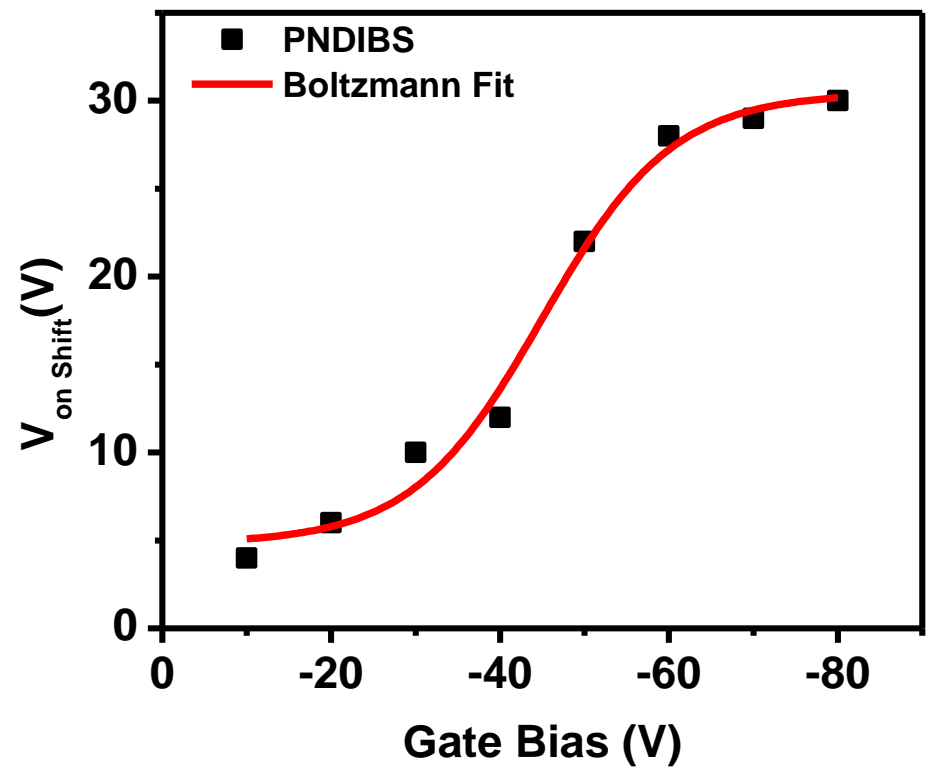

Figure 3. Shift in switching-on voltage with the applied gate bias voltage pulse for 1 second. The solid lines is a Boltzmann function fit. 

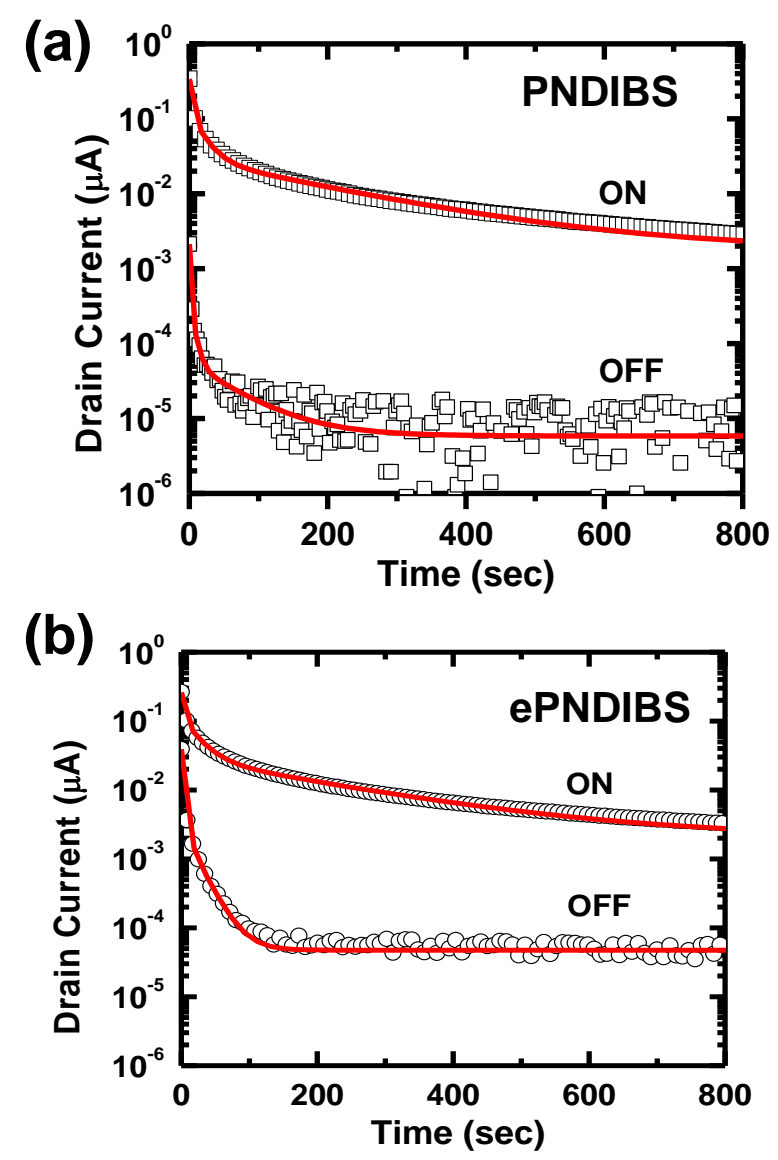

Figure 4. Retention (Write-Once-Read-Many-times; WORM) characterestics measured for (a) PNDIBS and (b) ePNDIBS OFETs after gate voltage $\left(\mathrm{V}_{\mathrm{gs}}= \pm 60 \mathrm{~V}\right.$ and $\left.0 \mathrm{~V}\right)$ pulses for 1 second and measured at $\mathrm{V}_{\mathrm{ds}}=40 \mathrm{~V}$. The solid lines are the fits of a stretched exponential equation with three time constants. 

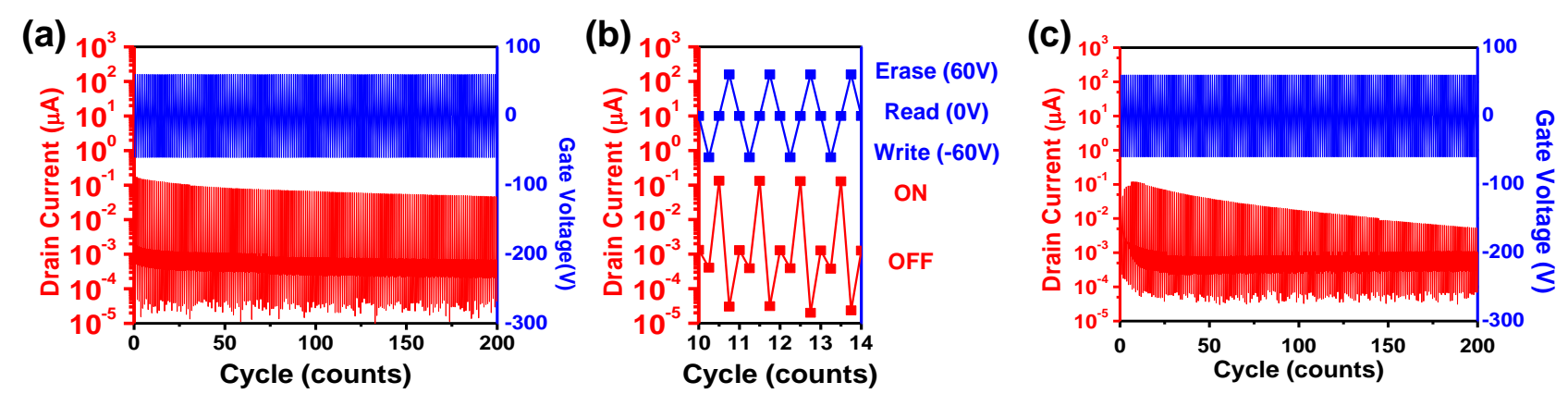

Figure 5. (a) Endurance (WRER) cycles of PNDIBS-OFET memory device, repeated over 200 cycles in 600 seconds: Writing biases were $\mathrm{V}_{\mathrm{gs}}=-60 \mathrm{~V}$ and $\mathrm{V}_{\mathrm{ds}}=0 \mathrm{~V}$; reading biases $\mathrm{V}_{\mathrm{gs}}=$ $0 \mathrm{~V}$ and $\mathrm{V}_{\mathrm{ds}}=40 \mathrm{~V}$; and erasing biases $\mathrm{V}_{\mathrm{gs}}=+60 \mathrm{~V}$ and $\mathrm{V}_{\mathrm{ds}}=0 \mathrm{~V}$. (b) The magnified plots of 4 endurance cycles after 10 cycles, clearly showing the difference between the written and erased states. (c) Endurance cycles of ePNDIBS-OFET memory device under the same conditions as in (a). 
(a)

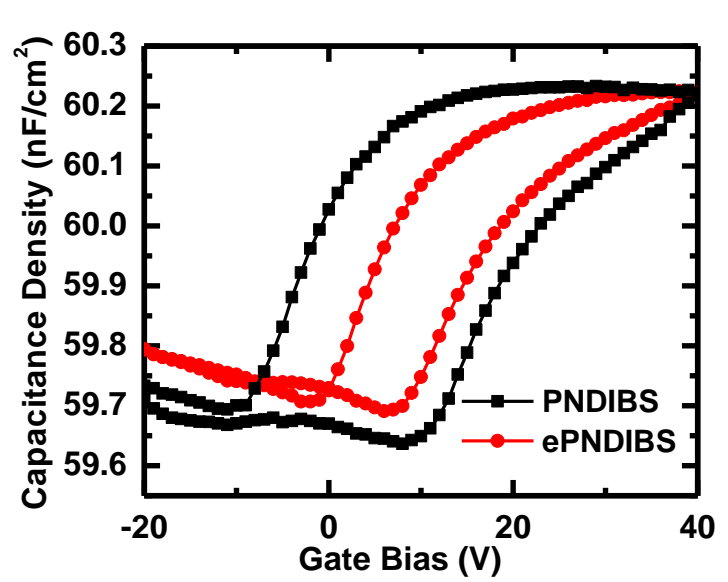

(b)

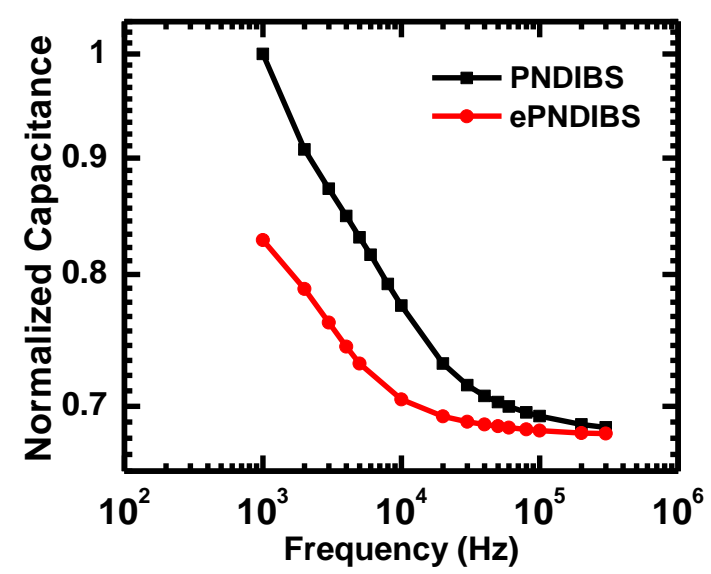

Figure 6. (a) Capacitance-voltage plot of PNDIBS-OFET and ePNDIBS-OFET obtained at 1 MHz frequency. (b) Normalized capacitance-frequency measured at the accumulation region $\left(\mathrm{V}_{\mathrm{gs}}=40 \mathrm{~V}\right)$. 


\section{Graphical Abstract}

\section{High-Performance Nonvolatile Memory Devices utilizing Charge-Trapping Phenomena in an n-Type Polymer Semiconductor}

Nishit M. Murari, ${ }^{1}$ Ye-Jin Hwang, ${ }^{1}$ Felix Sunjoo Kim, ${ }^{2, *}$ Samson A. Jenekhe ${ }^{1, *}$

1 Department of Chemical Engineering and Department of Chemistry, University of Washington, Seattle, Washington 98195-1750, United States

${ }^{2}$ School of Chemical Engineering and Materials Science, Chung-Ang University, Seoul, 156756, Republic of Korea
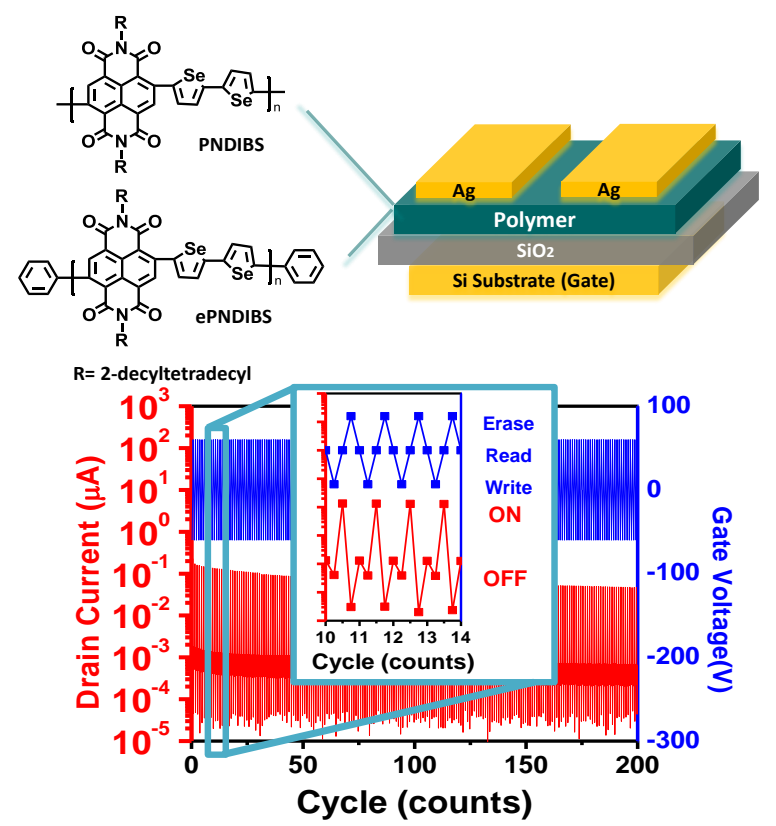